Original Research Paper

\title{
Performance Evaluation of an Assisting Device for Training "Normal-Like" Sit To Stand Movement
}

\author{
Pierluigi Rea, Erika Ottaviano and Gianni Castelli \\ Department of Civil and Mechanical Engineering, \\ University of Cassino and Southern Lazio, via G. Di Biasio 43, 03043, Cassino (FR), Italy
}

Article history

Received: 20-07-2017

Revised: 22-07-2017

Accepted: 25-07-2017

Corresponding Author: Erika Ottaviano

Department of Civil and Mechanical Engineering, University of Cassino and Southern Lazio, via G. Di Biasio 43, 03043, Cassino (FR), Italy

Email: ottaviano@unicas.it

\begin{abstract}
This paper presents a performance evaluation and results on the modelling and simulation of an assisting device to be used as aiding or guiding system for people with reduced mobility. There is a high level of motivation for elderly to perform independently basic Activities of Daily Life (ADL). Therefore, it is of great interest to design and implement reliable assisting devices that are able to help end-users mobility. Sit-To-Stand represent one of the most common ADL and a number of different devices have been proposed to reproduce a "normallike" movement. In general, a robot for a medical application should be able to interact with a patient in safe conditions, i.e., it must not damage people or surroundings; it must be designed to guarantee high accuracy and low accelerations during the operation. In addition, it should not be too bulky to interact closely with people. It can be advisable to have an easy-in-use and low-cost system, which can be used also in a domestic environment. In this study, a performance evaluation of a Sit-To-Stand assisting device is analyzed in terms of main characteristics, such as kinematic features and actuation requirements. Experimental tests were used to analyze a "normal-like" Sit-To-Stand movement and simulation results have been reported by considering a model of the human body interacting with the proposed assisting device.
\end{abstract}

Keywords: Assistive and Rehabilitation Engineering, Mechanical Design, Assisting Device, Simulation, Experimental Evaluation

\section{Introduction}

Recent studies have shown that a large number of elderly or people with motion disabilities, who stay at home, cannot perform Activities of Daily Life (ADL) without the aid of other people. Caregivers and nurses are usually involved, sometimes with external aid such as mechanical systems to lift the patients. The assisting devices are then developed to help an endusers and caregivers to perform ADL such as standing, walking and seating. More specifically, despite its apparent simplicity, Sit-To-Stand (STS) is a mechanically demanding functional task undertaken daily, requiring a strong coordination between posture and movement, but due to several reasons, it may represent a problem for elderly or people with reduced mobility. In addition, the STS is a crucial operation, since a person with reduced motion capability and physical strength can experience falling, which represents the most serious problem associated with aging. Assistive devices could enhance basic motor activities, such as postural changes, or more complex tasks, such as walk, leading to an improvement of the quality of life. Moreover, assistive devices could decrease fall incidence positively influencing health economics with the reduction in the high medical costs due to the traumatic consequences of falls.

Currently, in clinical practice in the STS training individuals extensively use their arms, while being additionally supported by one or two therapists (Burnfield et al., 2013). The movement is very slow as compared to normal STS movement as well as extremely physically challenging for therapists.

Some commercial solutions were developed and commercialized (Hari Krishnan and Pugazhenthi, 2014). Most of these devices act like end-effector robot, i.e., they provide assistive forces only at one body segment, while the trunk is either rigidly supported by a back support of a seat or through use 
of the arms. All these approaches may or surely result in unnatural kinematics.

Frequently, also the velocity of movement is rather limited, which does not correspond to the "normallike" STS transfer. A "normal-like" STS movement can be defined as a change of posture from the seated to standing ones obtained without external help. The trunk forward movement is compulsory at the beginning of motion to build sufficient momentum, which propels the $\mathrm{COM}$ in forward and vertical direction, followed by ascending motion facilitated by the extension of the joints of lower extremities (Hari Krishnan and Pugazhenthi, 2014).

The purpose of this work is to analyze main features of an assisting device designed for the STS.

More specifically, we developed a Sit-To-Stand trainer, which was designed to enable "normal-like" support of STS movement. Among several mechanical and mechatronic solutions developed by the authors, in this study we have chosen a 1-DOF device described in (Rea and Ottaviano, 2016). Secondly, we used a human body model to analyze main performances of the STS device in terms of kinematics characteristics such as velocity and acceleration profiles experienced by a point on the trunk of the human body model. In addition, experimental tests provided during a trial with adult healthy volunteers were used to obtain the requested trajectory of a point of interest experienced during a "normal-like" STS movement. That information, together with linear velocities of a point of interest of the body of an individual, were used to control the actuation during the simulation. Our aim was to examine whether a developed STS device is able to facilitate a "normallike" motion pattern of STS transfer, which is a prerequisite that such a device could be used in clinical rehabilitation practice.

Numerical results are reported for several cases of study dealing with a pre-defined laws of motion for the actuation and a motion profile of the actuators leading to a "normal-like" movement, which was obtained from experimental tests.

\section{Design issues on the Support Transfer Mechanism}

The STS movement requires a translation in two direction and rotation of the trunk, as described in (Marlene and Cooper, 1995). It can be defined as a balanced movement of the body's center of mass from a seated position to a standing one (Roebroeck et al., 1994) being a transition between two stable postures (Tully et al., 2005).

Several factors influence the STS, mainly the anthropometric data of the individual, age, environment, strategies (velocity, upper limb configuration) and objective (assistance, rehabilitation). Therefore, the design of the support mechanism, which is devoted to generate the requested motion and support the body of the individual during the STS is a crucial element in the design of the device.

It has been shown that a 1-DOF mechanism can reproduce a desired trajectory pattern, being defined according to a given set of data, or mean trajectory, mimicking a "natural-like" STS motion. For more advanced systems, 2-DOFs are in general required to accomplish any requested trajectory in the sagittal plane, if the orientation of the trunk has to be taken into account and additional DOF is required (Rea and Ottaviano, 2016). Spatial systems like parallel manipulators or cabledriven systems (Asker et al., 2015; Castelli and Ottaviano, 2010) are starting to be considered to design systems in the case of post stroke, to take into account asymmetries in the sagittal plane due to the limited motion of affected part of the body (Lomaglio and Eng, 2005).

In the last decades, robotic and mechatronic devices were successfully applied in the biomedical and biomechanical fields, providing some advantages over traditional therapies, such as objective measurements of the time-course of changes in motor control and semiautonomous practice of the exercise, (Marlene and Cooper, 1995). Although those concepts are related to rehabilitation techniques, they still hold for assisting devices. Devices for biomedical applications have as main purpose the interaction with humans, so they should be safe, reliable and possibly make slow movements (Rea et al., 2013a). According to the nature of the application, manipulators for medical applications must also meet the requirements of (Ottaviano, 2008):

- Reliability, to prevent and manage failures that may affect the safety of a patient

- Compactness and portability, to be used in even at home

- Simplicity, to be used by people without specific knowledge on Robotics

- $\quad$ Flexibility, to meet specific needs of each patient

According to these requirements, a mechatronic device can be designed and built allowing the use of the minimum number of DOFs to meet the requirements of simplicity in control, compactness and relatively lowcost. The mechanical design should fulfill the requirement of compactness. Flexibility can be pursued by using 2-DOFs in the sagittal plane to accomplish any desired trajectory, otherwise with 1-DOF mechanism the system should be synthesized adhoc. Mechanical solutions based on rigid links having 1, 2 or 3 DOFS can be used (Colombo et al., 2000; Tsukahara et al., 2009; Chuy et al., 2006), or systems based on cable-driven robots, as described in (Castelli and Ottaviano, 2010; Castelli et al., 2014). 
Since the large variability in parameters and solutions even in the early phase of type synthesis, i.e., definition of the type of mechanism to be used, it is of fundamental importance to be able to simulate correctly the system and make a performance analysis to compare main features of the system according to the large variation in the design solution.

\section{Human Biomechanical Model}

A simplified biomechanical model of the human in biomechanics and biomedical literature usually consists in a triple inverted pendulum, (Iqbal and Roy, 2004). A model consisting of five joints and six rigid bodies involving foot, lower leg (shank), upper leg (thigh), trunk (torso and head), lower and upper arm was considered in (Geravand et al., 2016).

In this study, we have considered a simplified human body model composed by 11 segments, which are connected by 10 revolute joints. Each segment is modeled by a relatively simple geometry that allows full body symmetry with respect to the sagittal plane (left right symmetry). Segments masses and dimensions have been assumed according to anthropometric data reported in (DINBelg, 2005) and summarized in Table 1. Each segment has been modeled with uniform density and center of mass being coincident with the center of volume.

The human body model in Fig. 1a was developed and tested for the simulation of the sit-to-stand, as shown in Fig. 1b. The simulation has been carried out in quasistatic condition and the speed of movement was set almost equal to the one used in the trial. During the simulation, the body model is seated on a wheelchair and then stand up with a natural movement without the interaction of the upper limbs, which are kept in a folded configuration to do not interact with the movement.

Figure 2 shows the trajectory of the point of interest placed on the shoulder of the body model. In the same plot, the orientation of the trunk is reported for some points of the trajectory, which is described in the sagittal plane (XY). The orientation of the trunk is given in degrees and measured with respect to the $\mathrm{X}$-axis.

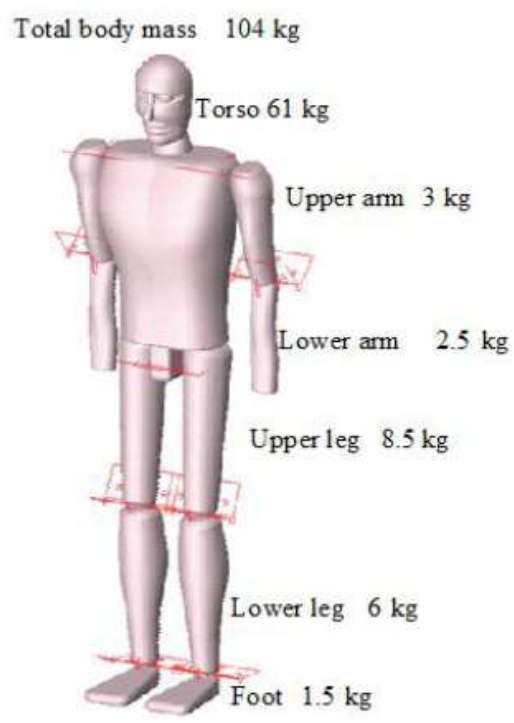

(a)

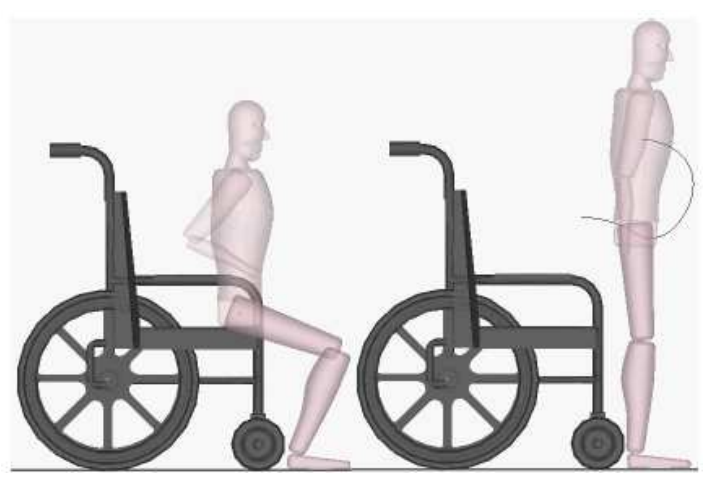

(b)

Fig. 1. A Model of human body: (a) Mass description; (b) Sit-To-Stand simulation 


\section{Experimental Evaluation of the "Normal-Like" Sit- To-Stand Movement}

Twenty healthy subjects took part in the experiment. Although the device is expected to be used by the elderly, healthy young subjects were chosen for their easier availability and reduced fatigue effects in the repeated execution of the STS task. As chair height was shown to influence the movement (Burdett et al., 1985), the chair height was set to $80 \%$ of the knee height of the subjects. Subjects were asked to sit on the chair such that their weight was supported only by the buttocks and not by the thighs. The feet were retracted toward the chair and the knee flexed so that the shank formed approximately 20 degrees with the vertical. The subject chose the distance between the feet freely. The arm were folded to not interact with the movement (the configuration was chosen freely by the subject). During the experimental trial, point trajectories and trunk orientation of were recorded and processed. Figure 3 shows experimental results on a male subject during the trial. In particular, Fig. 3a) reports the experimental set-up and Fig. 3b) gives the trajectory of a point on the shoulder, considered as fixed to the truck and some values of the trunk orientation along the trajectory. Figure 4 shows the trajectory of the reference point with indication of the time in seconds for the experiment reported in Fig. 3.

It is worth noting that we have reported an example in which the anthropometric data of the human body model are quite different from those of the volunteer to show the differences occurring with the same normallike movement. It is worth to note that the model has been validated when similar anthropometric data are used.

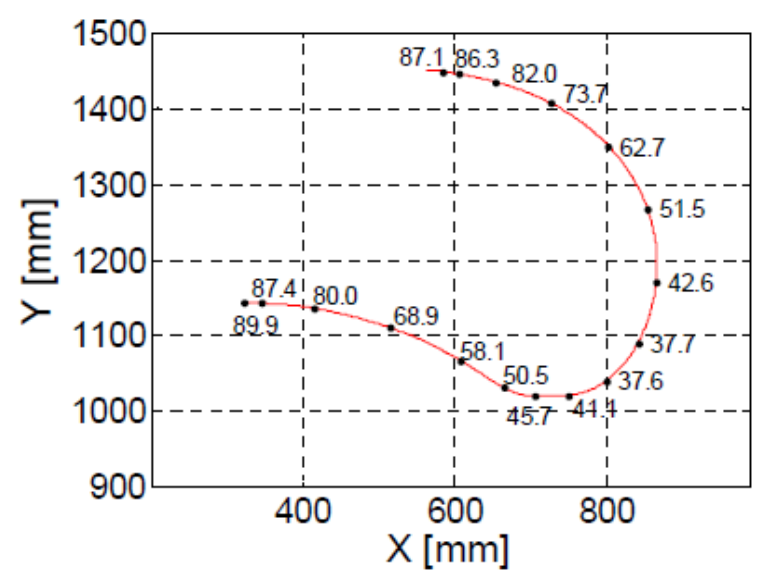

Fig. 2. Numerical results of the human body model during the Sit-To-Stand: Point trajectory with trunk orientation

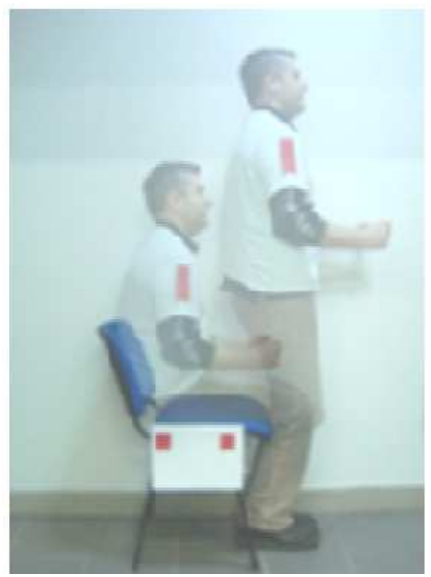

(a)

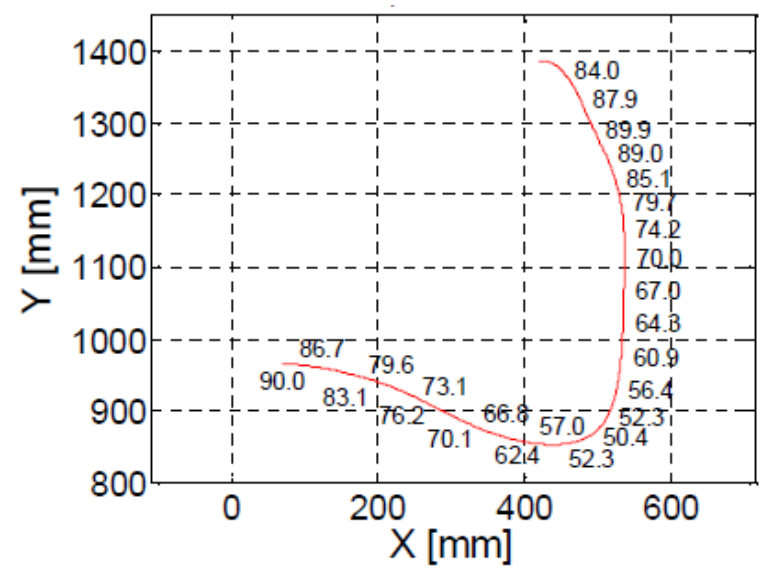

(b)

Fig. 3. Experimental results of a male subject during the Sit-To-Stand: (a) initial and final frames; (b) point trajectory and trunk orientation

Table 1. Selected anthropometric characteristics for the model of the human body

\begin{tabular}{lll}
\hline Body segments & Dimension $[\mathrm{mm} \times \mathrm{mm} \times \mathrm{mm}]$ & Mass [kg] \\
\hline Head + neck + torso + pelvis & $450 \times 890 \times 235$ & 61 \\
Upper arm & $110 \times 340 \times 110$ & 3 \\
Lower arm & $80 \times 300 \times 46$ & 2.5 \\
Upper leg & $23 \times 460 \times 23$ & 8.5 \\
Lower leg & $126 \times 440 \times 126$ & 6 \\
Foot & $131 \times 250 \times 60$ & 1.5 \\
Human body & $670 \times 1857 \times 315$ & 104 \\
\hline
\end{tabular}




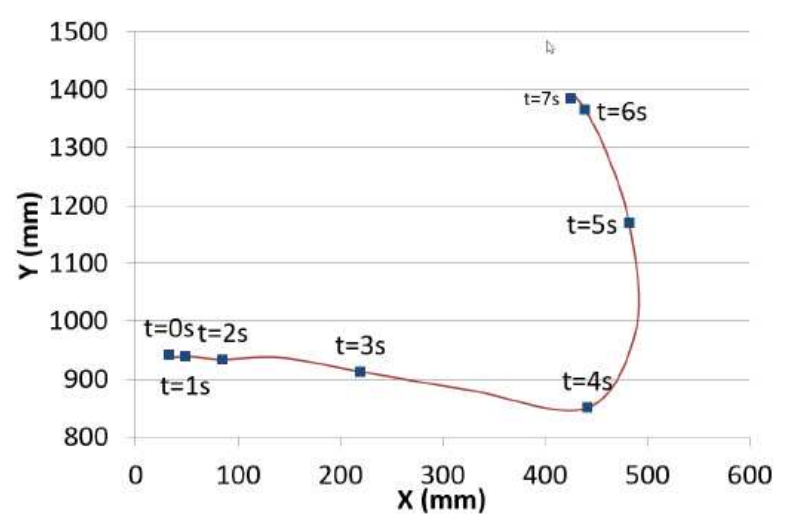

Fig. 4. Experimental results of a male subject in Fig. 3 during the Sit-To-Stand: Point trajectory and with indication of the time in seconds

\section{Performance Analysis}

Performance analysis was carried out by considering the following issues: The execution speed of the STS movement and stability verification according to the posture of the human body model interacting with the device. In particular, the simulation tests were performed by imposing a control law to the actuator or, alternatively, feeding to the system an actuator motion law to reproduce the "normal-like" STS movement obtained experimentally. Similar studies on performance evaluation were performed in (DallaLibera et al., 2016) analyzing the forces exchanged between the user and a device in the horizontal direction. The muscular activity was shown in (DallaLibera et al., 2016) to be lower in slow motion $(10-15 \mathrm{sec})$ than for speeds closer to the natural one (execution time of $3 \mathrm{sec}$ ).

In particular, it has been shown that the variation of forces is related to the acceleration experienced by the subject but different control strategies are used for different speeds (Pai et al., 1994).

In our work, we have studied the effects of the STS on the velocity of a point of interest, which is at the armpit. It will be shown that the law motion of the actuation greatly influence the resulting motion and it should be carefully planned in order to obtain a "natural like" STS motion.

\section{Simulation Results}

Experimental tests and numerical simulations were conducted and aimed at clarifying the effects of speed and motion law of the actuation for the execution of the STS movement supported by an assisting device. Among several designed solutions for the STS developed by the authors, e.g., (Castelli and Ottaviano, 2010; Rea et al., 2013b; Rea and Ottaviano, 2016) we have chosen to consider a 1-DOF mechanism for the simulation results.

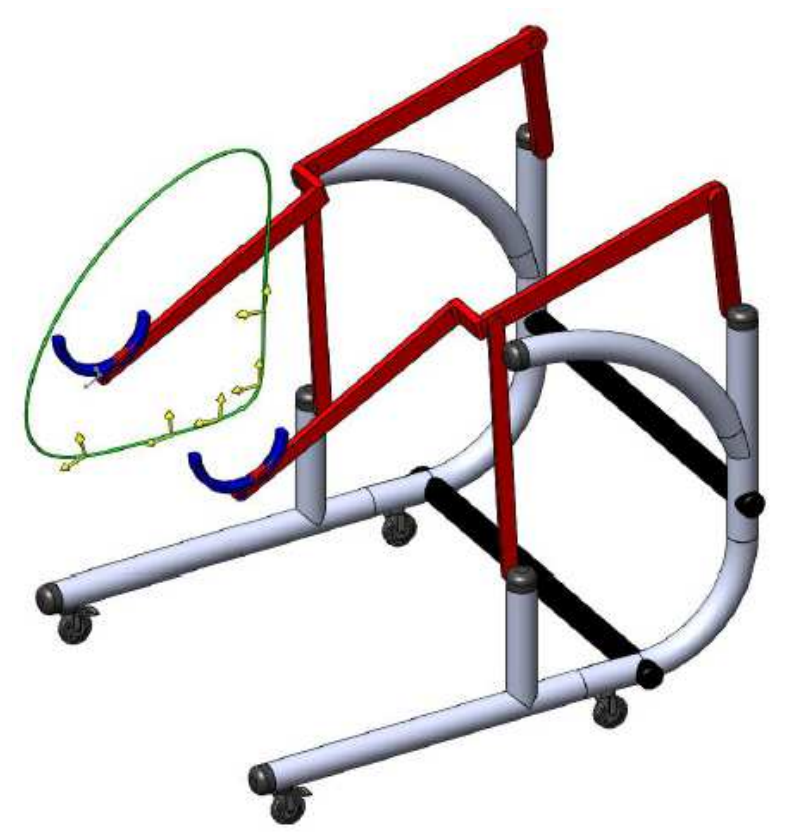

Fig. 5. 3D sketch of the 1-DOF mechanism for the generation of the trunk motion (Rea and Ottaviano, 2016)

The support mechanism is composed by a four-bar linkage, which has been synthesized ad-hoc for the proposed application (Rea and Ottaviano, 2016). The designed device that is shown in Fig. 5 provides a natural STS motion in terms of trajectory of the COM. The speed execution can be changed by operating the motor; in the reported simulations, we have considered several cases of study to analyze the effects in speed changes on velocities and accelerations of a point of interest on the human body model.

In particular, numerical simulations were carried out by using the developed human body model fixed to the device at the armpit, while feet are fixed to the ground. Figure 6 shows a motion sequence of the Sit-To-Stand motion of the human body model with the aid of the assisting device with 1-DOF shown in Fig. 5.

Simulation 1 was carried out considering a rotation of the input crank of $240 \mathrm{deg}$. and the duration of the movement was set equal to 5 seconds. In particular, Figure 7 shows the displacement of the input crank and its velocity profile. Figure 8 and 9 reports the linear velocity components for an armpit point and the acceleration components for the same point, taken as a reference fixed point.

Similarly, simulation 2 was carried out considering a constant velocity of the input crank of $8 \mathrm{rpm}$ and the duration of the movement was set to $5 \mathrm{sec}$, as shown in Fig. 10. The velocity and acceleration $\mathrm{X}$ and $\mathrm{Y}$ components for the simulation 2 are shown in Fig. 11 and 12 . 

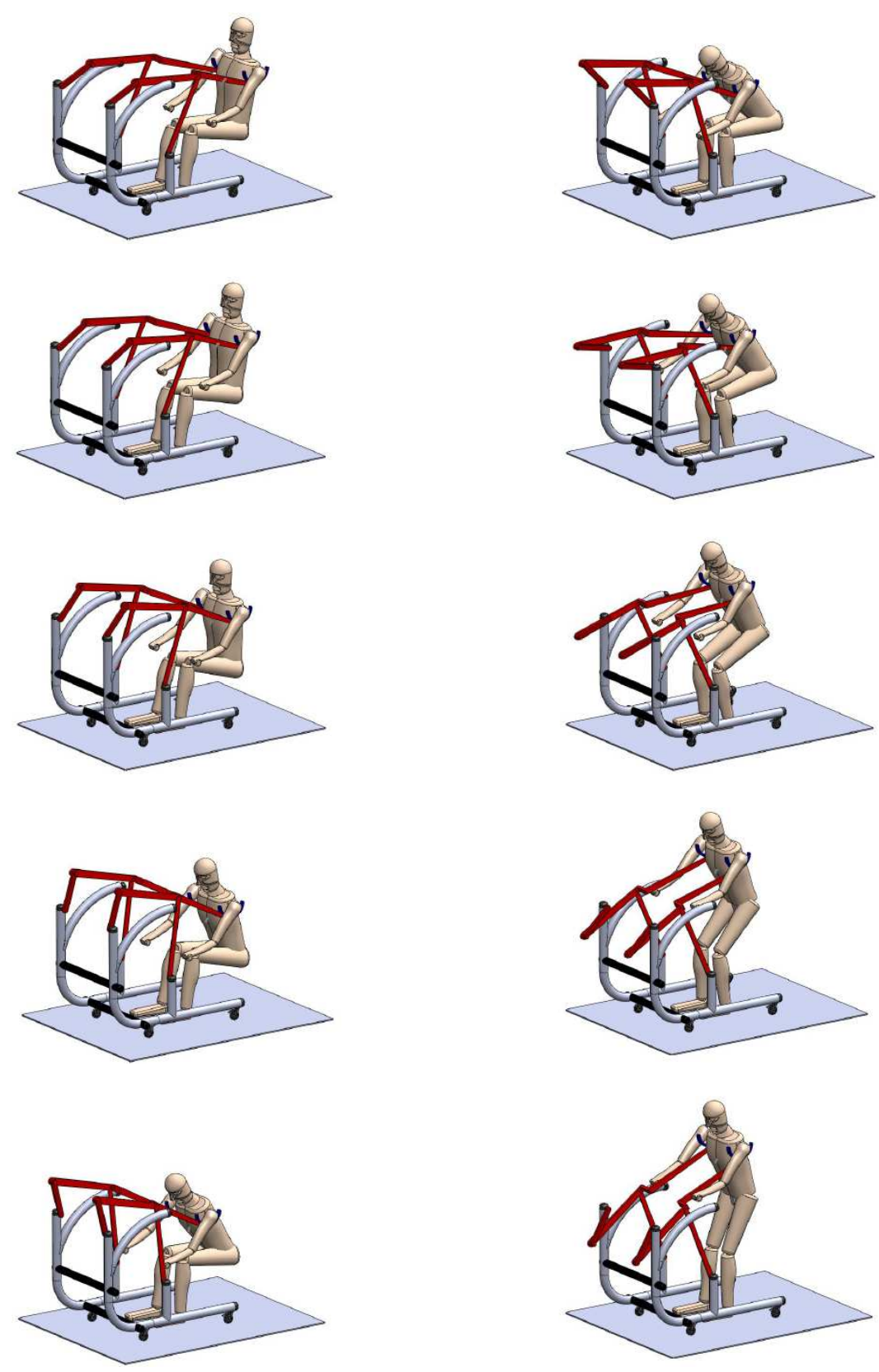

Fig. 6. Motion sequence of the Sit-To-Stand by using the 1-DOF device in Fig. 5



(a)

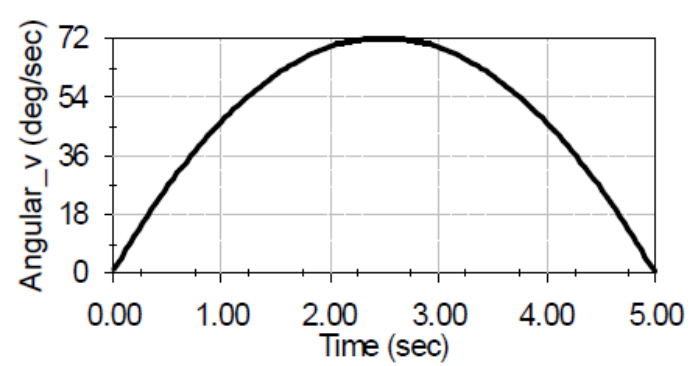

(b)

Fig. 7. Numerical results for simulation 1: (a) Angular displacement of the input crank; (b) velocity profile of the input crank 


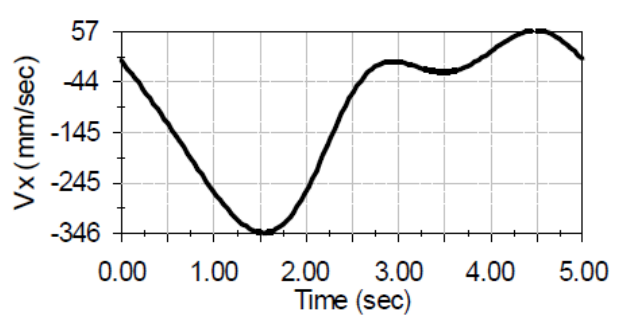

(a)

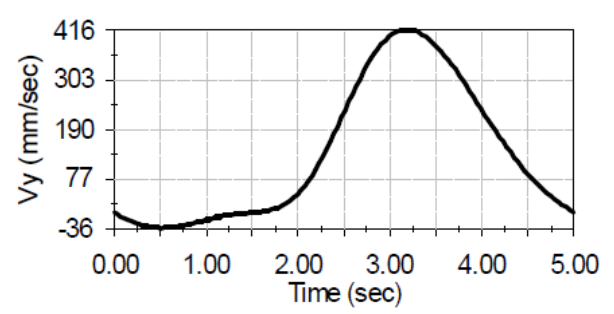

(b)

Fig. 8. Numerical results for simulation 1: (a) X-component Vx of the output velocity of an armpit point; (b) Y-component Vy of the output velocity of an armpit point

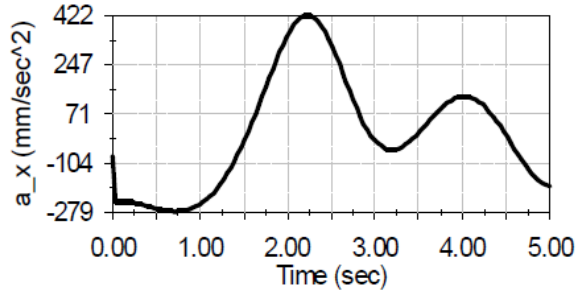

(a)

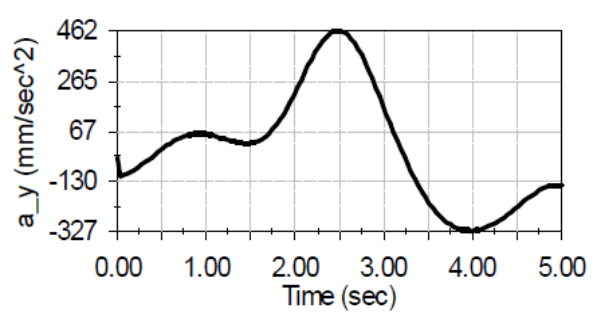

(b)

Fig. 9. Simulation 1: (a) X-component a_x and (b) Y-component a_y of the output accelerations of an armpit point

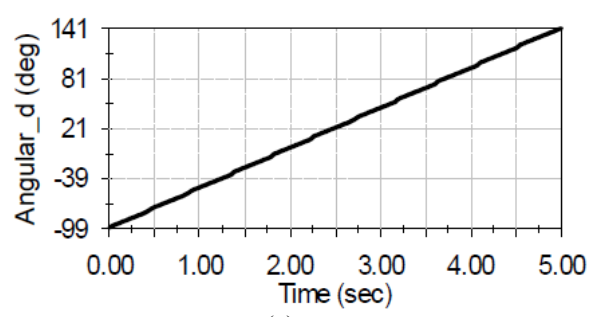

(a)

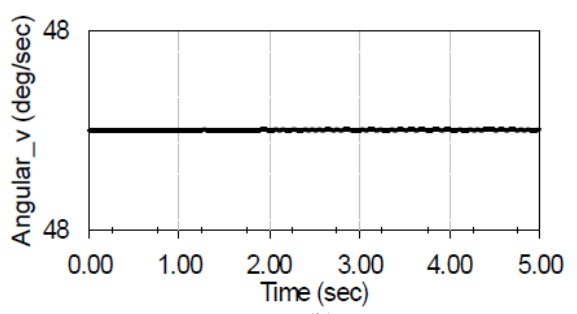

(b)

Fig. 10. Numerical results for simulation 2: (a) Angular displacement of the input crank; (b) constant velocity profile of the input crank

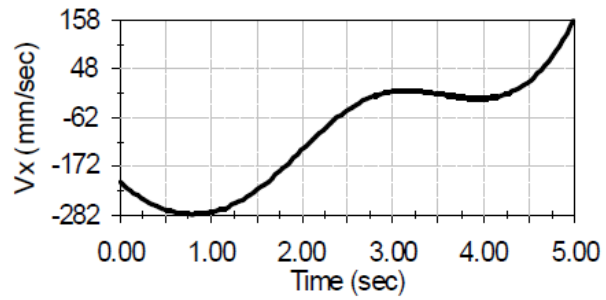

(a)

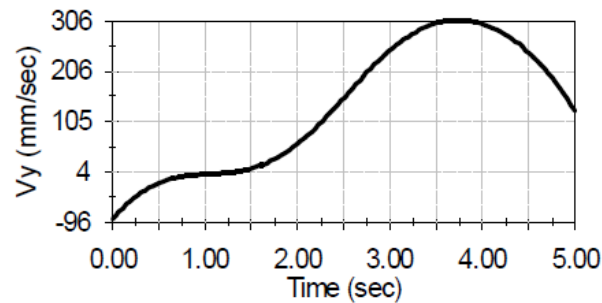

(b)

Fig. 11. Numerical results for simulation 2: (a) X-component Vx of the output velocity of an armpit point; (b) Y-component Vy of the output velocity of an armpit point

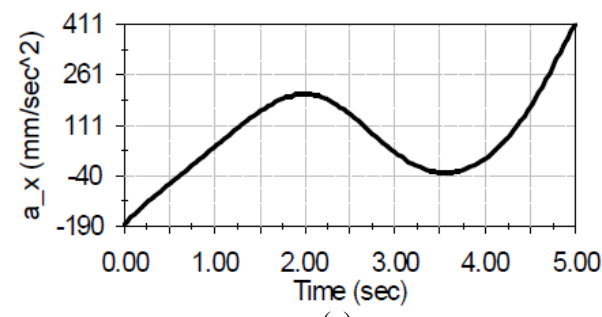

(a)

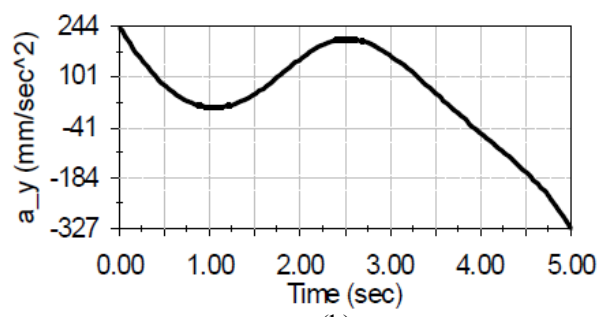

(b)

Fig. 12. Simulation 2: (a) X-component a_x and (b) Ycomponent a_y of the output accelerations of an armpit point 




(a)

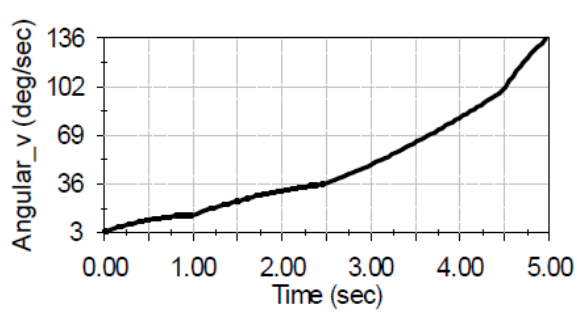

(b)

Fig. 13. Numerical results for simulation 3: (a) angular displacement of the input crank; (b) velocity profile of the input crank

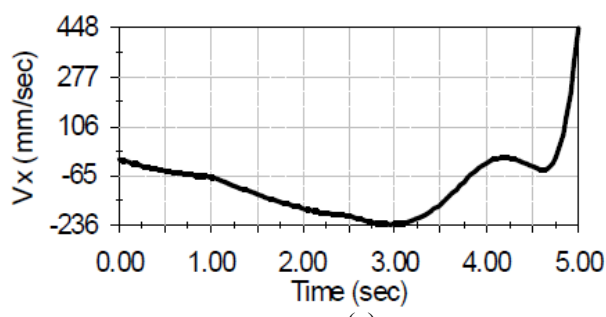

(a)

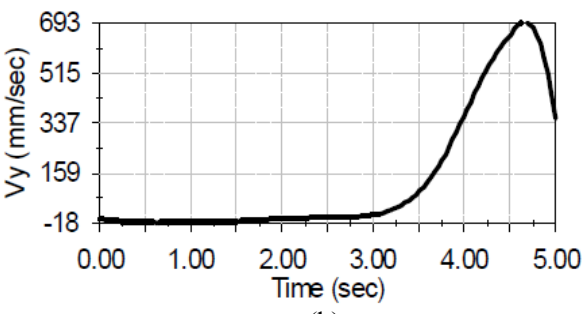

(b)

Fig. 14. Numerical results for simulation 3: (a) X-component Vx of the output velocity of an armpit point; (b) Y-component Vy of the output velocity of an armpit point

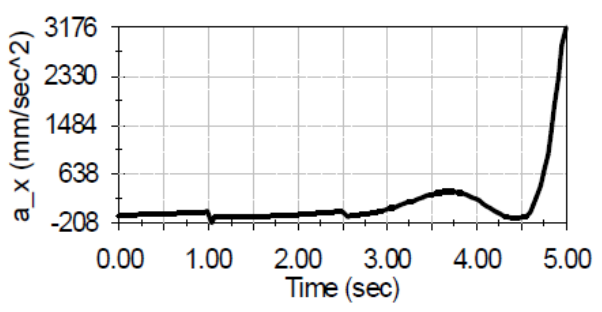

(a)

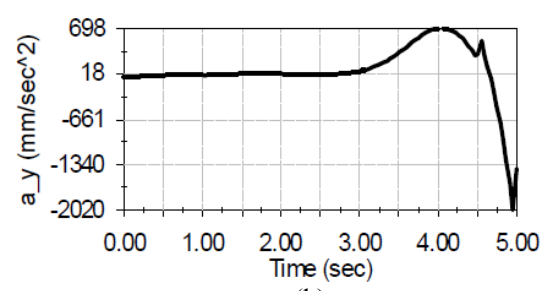

(b)

Fig. 15. Simulation 3: (a) X-component a_x and (b) Ycomponent a_y of the output accelerations of an armpit point

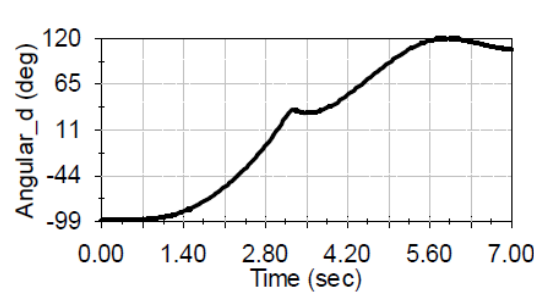

(a)

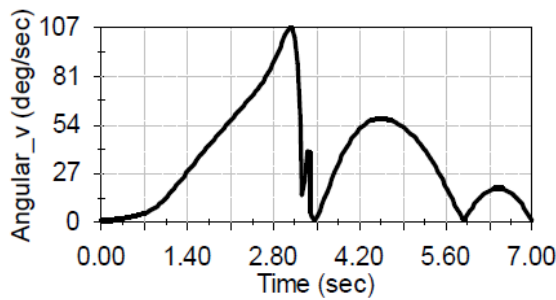

(b)

Fig. 16. Numerical results from the experimental trial in Fig. 3 to 5: (a) angular displacement of the input crank; (b) velocity profile of the input crank



(a)

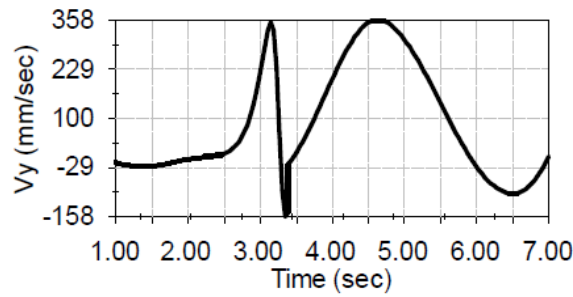

(b)

Fig. 17. Numerical results from the experimental trial in Fig. 3 to 5: (a) X-component Vx and (b) Y-component Vy of the output velocity of an armpit point 
Simulation 3 was carried out considering a constant velocity of the input crank of $8 \mathrm{rpm}$ and the duration of the movement was set to $5 \mathrm{sec}$. The velocity profile is given in Fig. 13b). Figure 14 and 15 report similar results to the previous cases.

Another kind of simulation test was considered, according to the experimental results obtained by the trials in Fig. 3 and 4 and considering a "natural-like" motion, in particular, the actuation was set in order to obtain kinematic features in terms of velocity and acceleration of a point of interest.

More specifically, the experimental results in Fig. 4 were used to simulate the motion of human body in terms of point velocity at the armpit and evaluate the law of motion and velocity of the input crank to get the requested kinematic features.

Figure 16 shows the angular displacement and the velocity profile of the input crank, which give the desired "natural-like" motion. Figure 17 shows the corresponding velocity components along $\mathrm{X}$ and $\mathrm{Y}$-axes, which are comparable with the experimental ones.

\section{Conclusion}

In this study, a performance evaluation of a Sit-ToStand assisting device is analyzed in terms of main kinematic characteristics. Experimental tests were used to obtain the so-called "normal-like" Sit-To-Stand movement and simulation results have been reported by considering a developed model of the human body interacting with a proposed mechanical device. The experimental data have been gathered to be used in the model to simulate a "natural-like" motion.

\section{Acknowledgement}

Adult volunteers that took part to the experimental trial at University of Cassino and Southern Lazio are greatly acknowledged.

\section{Funding Information}

The research leading to these results has received funding PRASG: University Research Projects "Starting Grant" from the University of Cassino and Southern Lazio. The first author would like to thank the Italian Ministry of Education, Universities and Research (MIUR) through the funded program PRASG to obtain the results of this research activity.

\section{Author's Contributions}

All the authors contributed equally to prepare, develop and carry out this work.

\section{Ethics}

This article is original. Author declares that are not ethical issues that may arise after the publication of this manuscript.

\section{References}

Asker, A., S.F. Assal and A. Mohamed, 2015. Dynamic analysis of a parallel manipulator-based multifunction mobility assistive device for elderly. Proceedings of the IEEE International Conference on In Systems, Man and Cybernetics, Oct. 9-12, IEEE Xplore Press, Kowloon, China, pp: 1409-1414. DOI: 10.1109/SMC.2015.250

Burdett, R.G., R. Habasevich, J. Pisciotta and S.R. Simon, 1985. Biomechanical comparison of rising from two types of chairs. Phys. Therapy, 65: 1177-1183. DOI: 10.1093/ptj/65.8.1177

Burnfield, J.M., B. McCrory, Y. Shu, T.W. Buster and A.P. Taylor et al., 2013. Comparative kinematic and electromyographic assessment of clinician- and device-assisted sit-to-stand transfers in patients with stroke. Phys. Therapy, 93: 1331-1341.

DOI: $10.2522 /$ ptj.20120500

Castelli, G. and E. Ottaviano, 2010. Modelling, simulation and testing of a reconfigurable cablebased parallel manipulator as motion aiding system. Applied Bion. Biomechan., 7: 253-268.

DOI: $10.1080 / 11762322.2010 .512733$

Castelli G., E. Ottaviano and P. Rea, 2014. A Cartesian Cable-Suspended Robot for improving end-users' mobility in an urban environment. Robot. Comput.Integrated Manuf., 30: 335-343.

DOI: $10.1016 /$ j.rcim.2013.11.001

Chuy, O., Y. Hirata, Z. Wang and K. Kosuge, 2006. Approach in assisting a sit-to-stand movement using robotic walking support system. Proceedings of the IEEE/RSJ International Conference on Intelligent Robots and Systems, Oct. 9-15, IEEE Xpore Press, Beijing, China, pp: 4343-4348.

DOI: 10.1109/IROS.2006.282007

Colombo, G., M. Joerg, R. Schreier and V. Dietz, 2000. Treadmill training of paraplegic patients using a robotic orthosis. J. Rehabilit. Res. Dev., 37: 693-700. PMID: 11321005

DallaLibera, F., Y. Tsusaka, Y. Okazaki, R. Futakuchi and M. Yamamoto et al., 2016. Analysis of velocity's influence on forces and muscular activity in the context of sit-to-stand motion assisted by an elderly care robot. Proceedings of the IEEE/RSJ International Conference on Intelligent Robots and Systems, IEEE Xplore Press, Daejeon, South Korea, pp: 717-724. DOI: 10.1109/IROS.2016.7759131 
DINBelg, 2005. http://www.dinbelg.be/DINBelg\%202005\%20antrop ometrie\%20tabel.PDF

Geravand, M., P.Z. Korondi, C. Werner, K. Hauer and A. Peer, 2016. Human sit-to-stand transfer modeling towards intuitive and biologically-inspired robot assistance. Autonomous Robots, 41: 575-592. DOI: $10.1007 / \mathrm{s} 10514-016-9553-5$

Hari Krishnan, R. and S. Pugazhenthi, 2014. Mobility assistive devices and self-transfer robotic systems for elderly, a review. Intell. Service Robot., 7: 37-49. DOI: $10.1007 /$ s 11370-013-0142-6

Iqbal, K. and A. Roy, 2004. Stabilizing PID controllers for a single-link biomechanical model with position, velocity and force feedback. ASME Trans. Biomech. Eng., 126: 838-840. DOI: 10.1115/1.1824134

Lomaglio, M.J. and J.J. Eng, 2005. Muscle strength and weight-bearing symmetry relate to sit-to-stand performance in individuals with stroke. Gait Posture 22: 126-131. DOI: 10.1016/j.gaitpost.2004.08.002

Marlene, J.A. and J. Cooper, 1995. Biomechanics of Human Movement. 2nd Edn., WCB, Brown and Benchmark, Madison, ISBN-10: 007115177X, pp: 572.

Ottaviano, E., 2008. Design issues and application of cable-based parallel manipulators for rehabilitation therapy. Applied Bion. Biomech., 5: 65-75.

DOI: $10.1080 / 11762320802373701$

Pai, Y.C., B. Naughton, R. Chang and M. Rogers, 1994. Control of body centre of mass momentum during sit-to-stand among young and elderly adults. Gait Posture, 2: 109-116.

DOI: 10.1016/0966-6362(94)90100-7
Rea, P. and E. Ottaviano, 2016. Analysis and mechanical design solutions for sit-to-stand assisting devices. Am. J. Eng. Applied Sci., 9: 1134-1143. DOI: 10.3844/ajeassp.2016.1134.1143

Rea, P., E. Ottaviano and G. Castelli, 2013a. A procedure for the design of novel assisting devices for the sit-to-stand. J. Bionic Eng., 10: 488-496. DOI: $10.1016 /$ S1672-6529(13)60249-8

Rea P., E. Ottaviano, M. Conte, A. D'Aguanno and D. De Carolis, 2013b. The design of a novel tilt seat for inversion therapy. Int. J. Imag. Robot., 11: 1-10.

Roebroeck, M.E., C.A.M. Doorenbosch, J. Harlaar, R. Jacobs and G.J. Lankhorst, 1994. Biomechanics and muscular activity during sit-to-stand transfer. Clin. Biomech., 9: 235-244. DOI: 10.1016/0268-0033(94)90004-3

Tsukahara, A., Y. Hasegawa and Y. Sankai, 2009. Standing-up motion support for paraplegic patient with Robot Suit HAL. Proceedings of the IEEE International Conference on Rehabilitation Robotics, IEEE Xplore Press, Kyoto, Japan, pp: 211-217. DOI: 10.1109/ICORR.2009.5209567

Tully, E.A., M.R. Fotoohabadi and M.P. Galea, 2005. Sagittal spine and lower limb movement during sit-to-stand in healthy young subjects. Gait Posture, 22: 338-345.

DOI: $10.1016 /$ j.gaitpost.2004.11.007 